\title{
BreathID: Radar's New Role in Biometrics
}

This paper was downloaded from TechRxiv (https://www.techrxiv.org).

\section{LICENSE}

CC BY 4.0

SUBMISSION DATE / POSTED DATE

06-09-2021 / 09-09-2021

CITATION

Islam, Shekh Md Mahmudul (2021): BreathID: Radar's New Role in Biometrics. TechRxiv. Preprint. https://doi.org/10.36227/techrxiv.16574264.v1

$\mathrm{DOI}$

10.36227/techrxiv.16574264.v1 


\title{
BreathID: Radar's New Role in Biometrics
}

\author{
Shekh M. M. Islam, Member, IEEE and Victor M. Lubecke, Fellow, IEEE
}

The word "Radar" reminds us of the battlefield, and it is typically associated with military applications. Radar has been used in a widespread area of applications, including weather forecasting, vehicle speed measurement, detection, and the presence of aircraft, ships, and so on [1]. However, over the past four decades, there has been an increasing interest in modifying this battlefield tool into healthcare applications such as detecting the breathing rate and heart rate of humans unobtrusively (without attaching any sensor to the chest surface) [2]. Emerging applications of radar sensing also include automotive radar for driverless car navigation, portable remote healthcare monitoring (sleep position recognition, sleep quality and status, etc.), smartphone hand gesture recognition, and human activity recognition.

Radar is gaining attention from a wide audience of students in electrical and computer engineering, researchers, academics, entrepreneurs, and policymakers due to its suitability for a wide area of applications. There is also a strong preference for the contactless nature of this sensing technology for subject detection applications, especially for search and rescue and surveillance operations for post-disaster management. Different clinical investigations have proven that breathing dynamics or heart-based cardiac cycle mechanisms are unique for different subjects [3]. Radar is also an attractive approach for remote respiration sensing as it is a non-contact and unobtrusive form of measurement [4]. Cardiac dynamic parameters/features can also be extracted using radar technology and it is also possible to unobtrusively authenticate the identity of individuals with the advent of advanced pattern recognition and machine learning technologies.

This article is focused on providing some insight into the requirements for a non-contact continuous identity authentication system using radar sensing technologies. The article also discusses the basic principles supporting the potential new role of radar sensing technologies in a broad area of applications. It also 
highlights challenges associated with this new technology and provides some direction on what is needed to address requirements for real-world applications.

\section{Need for a new unobtrusive continuous identity authentication system}

Interest in the adoption of non-contact radar sensing technologies for biometric identification has arisen from the need to reduce the vulnerability of traditional identity authentication systems (fingerprint, password, or facial recognition). Typical conventional identity authentication systems rely on one-pass validation which means the user is only subjected to an initial check at the start of a user session, after which malicious system adversaries might take over and hack personal information, such as bank account numbers, social security number, and personal account information [5]. Continuous identity authentication can greatly increase security, yet such systems typically have significant limitations such as users needing to intentionally engage with the authentication system repeatedly using a password or fingerprint which can disrupt their concentration and interfere with normal work and activities. In 2019 more than 3.2 million identity fraud cases (mostly credit card, social security, and social account fraud) were reported to the Federal trade commission (FTC) of the United States [5]. Most of these malicious system attacks took advantage of the one-pass validation nature of the traditional identity authentication systems and user carelessness [5].

Behavioral biometric-based authentication has been investigated which relies on the recognition of patterns such as a keystroke or gaze dynamics. However, keystroke dynamics recognition requires the user to continuously stroke the keyboard while gaze pattern recognition requires the user to continuously face and look at the screen [6]. Other methods involving facial recognition using a video camera can introduce privacy issues. Identity recognition through physiological signals like electrocardiogram (ECG) from heart signals and Electroencephalogram (EEG) from brain waves have also been examined [6]. However, such physiological-based attempts typically require a user to continuously wear sensors on their body surface which is uncomfortable which can make the approach impractical [6]. Thus, a non-contact, unobtrusive continuous user authentication scheme via non-contact respiration sensing would be a significant 
improvement that has the potential to reduce vulnerability to malicious attacks and also increase usability in practice [6].

\section{Advantages of radar-based respiration sensing for non-contact identity authentication}

What can radar-based respiration sensing technology contribute to biometric identification systems? To answer this question, the potential advantages of radar over other sensor technologies will be described first followed by an explanation of how respiration patterns can be a unique biometric feature. Radar research in healthcare has primarily focused on extracting breathing rate and heart rate without attaching any sensors to the body $[7,8]$. Before describing the details of the basics of non-contact respiration sensing, it is worth exploring why in recent years radar rather than other technologies has received more attention for physiological sensing. Radar is an attractive approach as it is non-contact and unobtrusive, so users need not wear any sensors on the body and they need not intentionally engage themselves with the system $[7,8]$. Radar sensor performance does not depend on good lighting and can even work through clothing or bedding as well as other visual obstructions [7]. Most importantly, in terms of perceived privacy Radar is far preferable to installing video cameras in a house [8]. Any type of camera can be regarded as privacy-invasive. No intrusive video imaging is required even to recognize people, as the level of radar feature recognition provided is very rich and will be discussed in a later section. A related topic of interest involves addressing the cost and installation of the radar in the home environment as there is not yet mass-produced equipment available for radar use in healthcare. However, there is a range of emerging mass-produced technology for the autonomous vehicle market and automotive radar may provide the push to make healthcare radar accessible. Thus the key research remaining is that needed for the recognition of breathing patterns or heart-based dynamics which are important and unique features needed for distinguishing between different human beings.

Breathing is a complicated anatomical process that takes place through the control of the central neural mechanism in the coordination of lung, diaphragm, and respiratory muscles $[3,6,8,9]$. In general, the breathing mechanism has three different stages: inhale, exhale, and a pause in between [10]. Different clinical investigations proved that for awake adult human subjects at rest, there exists diversity in breathing patterns 
not only in terms of tidal volume, and inspiratory and expiratory duration, but also in airflow profile [3]. Everyone selects for one pattern among the number of infinite possible ventilatory variables and airflow profiles. This variability is non-random and may be explained by either a central neural mechanism or by the instability in the chemical feedback loops [3]. In addition, heart-based geometry is different for different subjects and clinical investigation also suggests that cardiac cycle variations occur based on different shapes of the heart [6]. Radar can detect respiratory motion and it is also possible to extract unique features based on diversity in the breathing dynamics.

\section{Basic Principle of non-contact radar respiration sensing technology}

Radar stands for radio detection and ranging [1]. Radar uses transceivers that have both transmitter and receiver capability [1]. A radar transmits an electromagnetic signal and collects the reflection of that signal from a target and analyzes the waveform to extract the velocity, distance, and position [4]. It can be compared with echolocation commonly associated with animals such as bats or dolphins, which transmit an ultrasound wave and get information about prey from the reflected wave. A radar can have two different antennas for transmission and reception or may have a single antenna for both functions. Radar for healthcare monitoring also works in different frequency ranges from $2.4 \mathrm{GHz}$ to $24 \mathrm{GHz}$ (within the Industrial, the scientific, and Medical band (ISM)). Radar also incorporates a digital signal processing core where data can be filtered and stored for further processing. Commonly used radar can be classified into two different systems, one of which is continuous wave $(\mathrm{CW})$ radar which sends an unmodulated (same carrier frequency) signal, and another one which is frequency modulated (different carrier frequencies) continuous wave (FMCW) radar which sends a modulated signal [7]. FMCW sends a modulated signal so it can determine the absolute range of the target without ambiguity. In this article, CW radar is mainly described because in healthcare sensing we need to know the relative chest displacement and $\mathrm{CW}$ radar can do this with simple circuitry and is less expensive than FMCW radar [7]. For physiological sensing, the basic principle used by radar is the Doppler effect. According to the Doppler principle when a signal reflects from a moving chest surface its phase information changes [7]. The phase change of the reflected signal is directly proportional to the tiny movement of the 
chest surface due to cardio-respiratory activities [7]. Figure 1 illustrates the basic principle of respiration sensing technology using radar where the reflected signal phase is different from the transmitted signal. After the acquisition of the reflected signal, a typical $\mathrm{CW}$ radar module multiplies that signal with the transmitted signal and then filters out components of interest. In Doppler radar respiration sensing technology, radar received echoes contain both heartbeat and respiration motion modulations that are superimposed on each other [8]. Because the chest moves a much greater distance due to breathing than it does due to the heart movement, the respiration rate can be detected without filtering. To detect the heart rate, the smaller heart signal must be separated from the respiration signal [8]. In general, the heart and respiration signals are isolated in frequency bands although include overlapping harmonics. For a human subject in a sedentary condition, the heart rate is normally between 0.83 and $1.5 \mathrm{~Hz}$ (50 to 90 beats per minute) while the breathing rate is between 0.15 to $0.4 \mathrm{~Hz}$ (9 to 24 breaths per minute) [3]. Although heart rate can vary from 43 to 94 beats per minute $(0.7$ to $1.6 \mathrm{~Hz})$ and breathing rate from 5 to 21 breaths per minute $(0.08$ to $0.35 \mathrm{~Hz})$ depending on the physiology of different subjects [3]. Thus, a high pass filter between $0.70 \mathrm{~Hz}$ to $0.35 \mathrm{~Hz}$ is
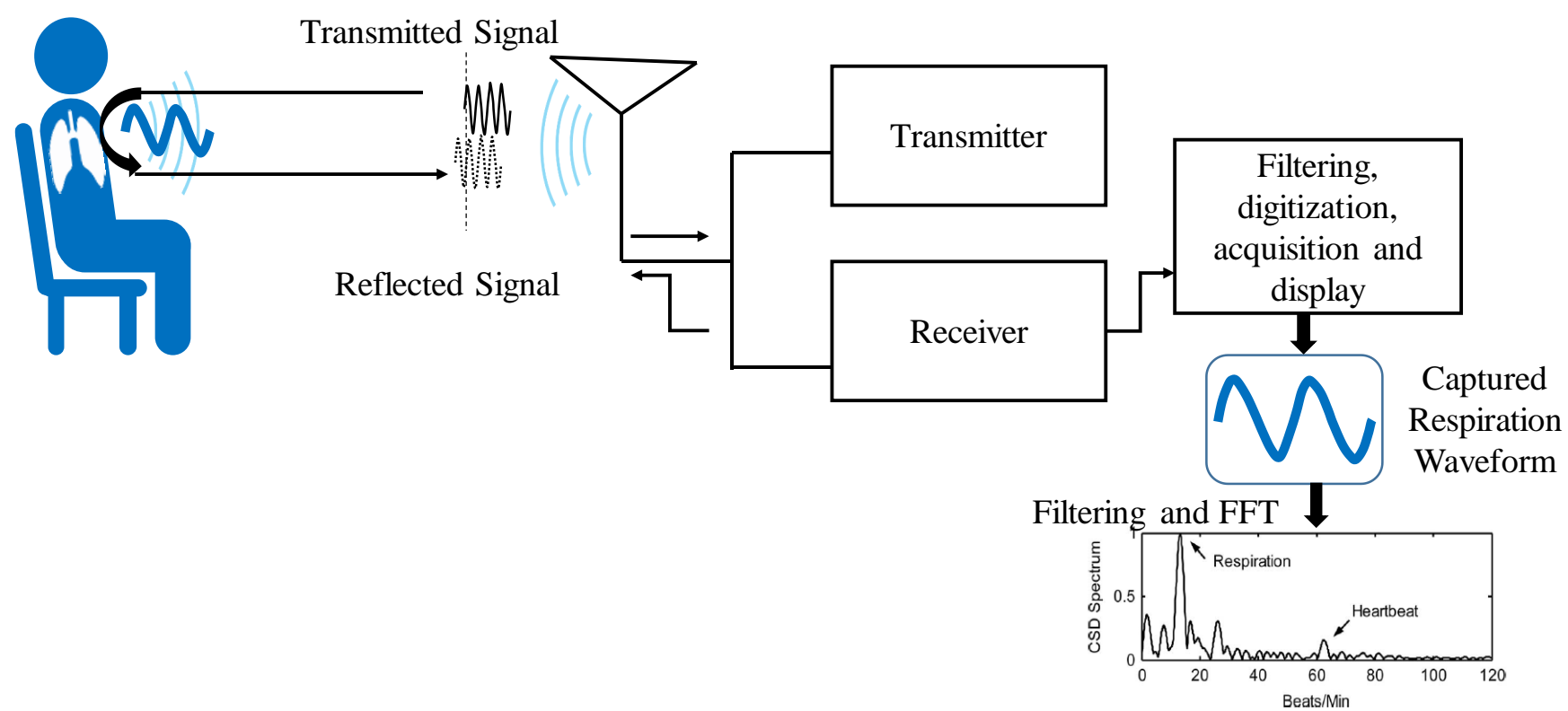

Fig. 1. Basics of Doppler CW radar physiological sensing. The phase of the reflected signal changes in direct proportion to the tiny movement of the chest surface caused by cardio-respiratory activity. Filtering and demodulation take place at the receiver before further processing. After band-pass filtering and FFT analysis, dominant frequencies are isolated, corresponding to breathing rate and heart rate. The problem is complicated by the fact that breathing rate and heart rate frequencies generally contain overlapping harmonics. Additional signal processing is also typically needed to separate the ohysiological motion of interest from extraneous motion. 
used to isolate the heart signal from the respiration signal [8]. Post-processing can be conveniently performed using MATLAB, a popular software tool, to extract breathing rate and heart rate after isolating the heart signal from the respiration signal. For extracting breathing rate and heart rate, the Fast Fourier transform (FFT) is utilized to convert the time-domain signal into a frequency domain signal where the dominant peaks breathing rate and heart rate can be resolved [7]. In practical scenarios, additional signal processing is needed for suppressing extraneous motion in the field of view of the radar. In this way, respiratory information can be extracted unobtrusively.

\section{The radar-based non-contact identity authentication system}

Recently, radar-based identity authentication systems are gaining attention as many researchers investigate

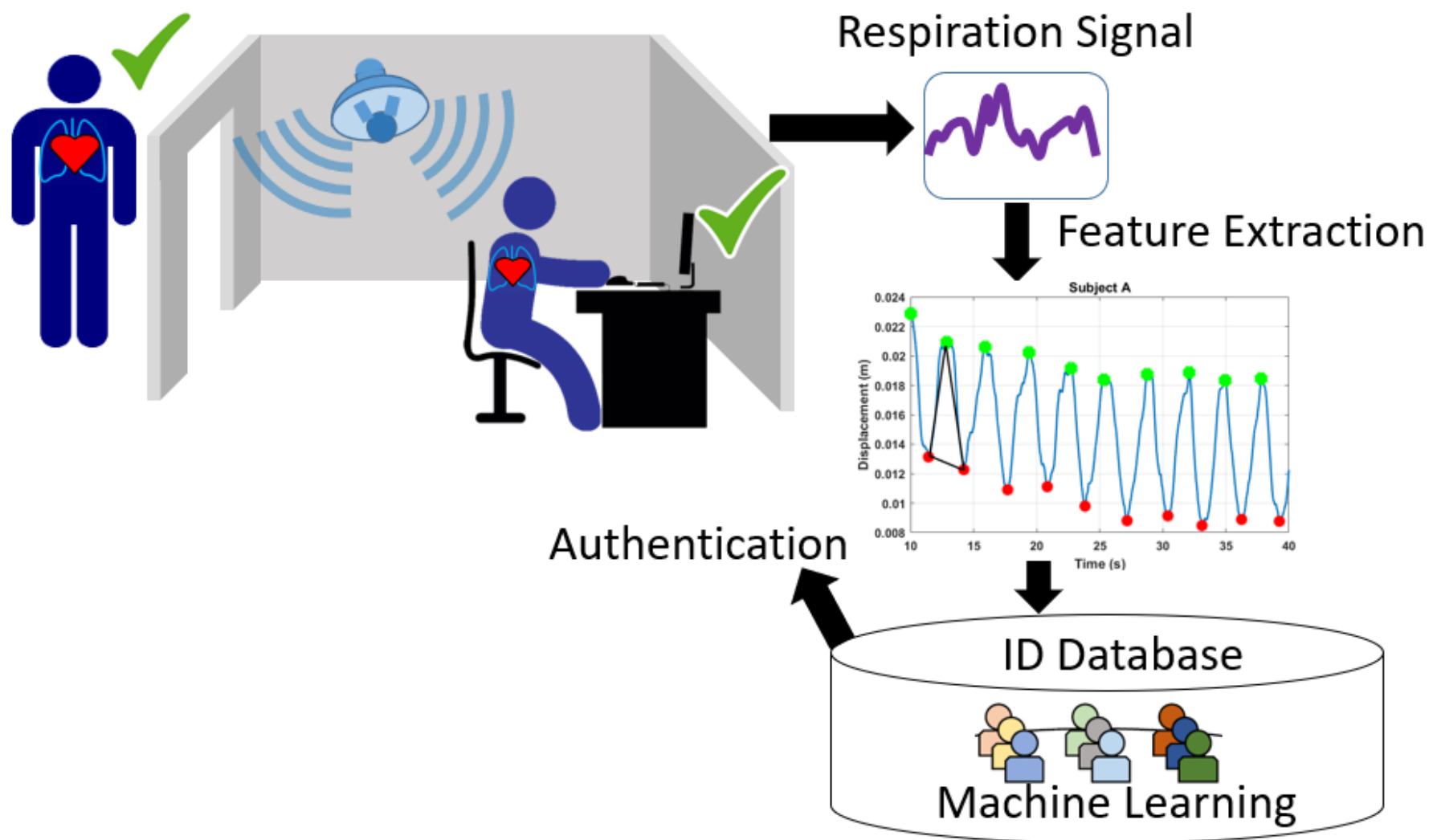

Fig. 2. Proposed Doppler-radar based non-contact continuous identity authentication system. The human respiration signal pattern is captured by radar, and features are extracted using a unique identification algorithm and machine learning classifiers. Different participants are recognized based on their distinct respiratory features. Based on [11].

the feasibility of this technology, with efficacy already demonstrated $[9,10]$. Based on reported results 
researchers have focused on extracting two different measures: respiratory-based and heart-based features [11]. The Biosensing Laboratory at the University of Hawaii at Manoa focused on a respiratory-based featurerelated identity authentication system $[10,11,12,13,14]$, which is described here in-detail along with the feasibility of the use of heart-based features in recognizing people [11]. An illustration of a proposed unobtrusive biometric identification system based on respiration is shown in Figure 2. The methodology can be broken down into four parts:

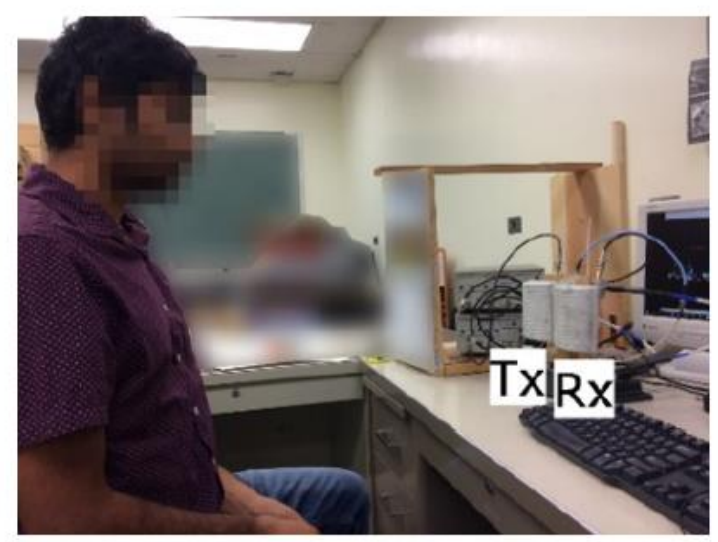

(a)

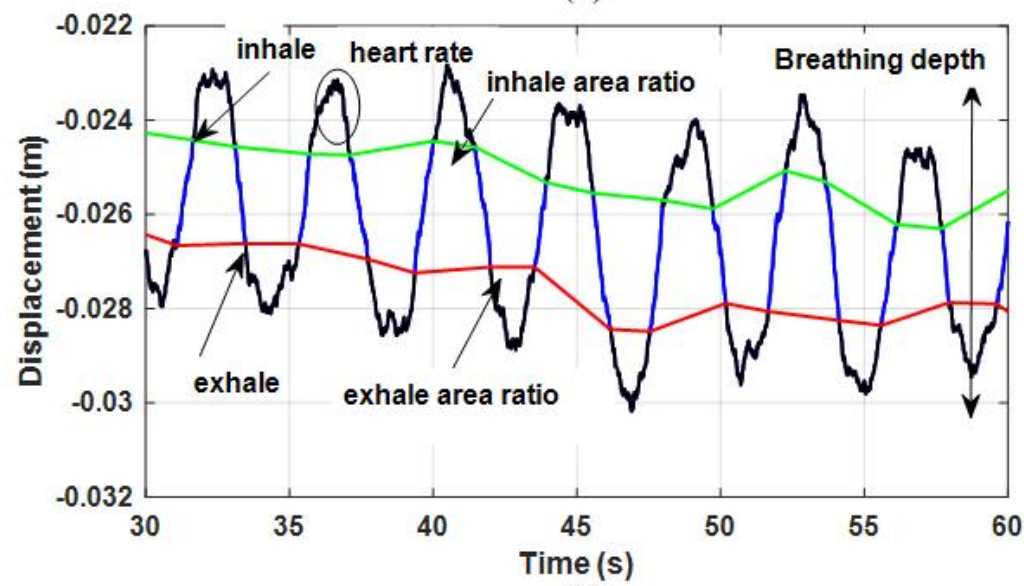

(b)

Fig. 3. Unique feature extraction. Measurement setup with transmission (Tx) and receiving (Rx) antennas shown for a radar transceiver with a test participant seated on a chair (a). After capturing the respiration pattern, different respiratory related dynamic features were extracted for 20 participants. In this feature list inhale rate, exhale rate, heart rate, inhale area, exhale area, inhale/exhale area ratio, and breathing depth were extracted. From [12].

(1) signal acquisition, (2) pre-processing, (3) feature extraction, and (4) machine learning (ML) classifier integration. A 2.4-GHz Doppler radar system was used to capture the respiration pattern for twenty different 
test participants over a course of about two months [11,12,13,14]. After capturing the respiration pattern, the signals were processed for removal of noise and another random movement, and then respiratory-related features were extracted and used to recognize different participants. For feature extraction, a dynamic segmentation technique was used where $30 \%-70 \%$ of the amplitude of the signal was used, and from within that segment, the area between inhale and exhale episodes was found for about 60 s of respiration cycle captures [10]. Different people had different inhale and exhale area ratios which indicates how the first breath initiates the next cycle of breathing $[10,11]$. Different people have different pause times in starting the next cycle of breathing pattern and there are significant differences in air flow profile after performing physiological activities (walking upstairs) [12]. Different respiratory features like breathing rate, heart rate, inhale rate, exhale rate, inhale area, exhale area, inhale/exhale area ratio were also extracted for the twenty different participants $[12,13,14]$. Figure 3 shows the experimental scenario performed at the Biosensing Laboratory of the University of Hawaii at Manoa, where experiments were conducted. All respiratory dynamics-related features were extracted and fed into a machine learning classifier to recognize participants. A training data set and test data set were separated for both sedentary breathing and after physiological activities (walking upstairs) [12,13,14]. Three different popular machine learning classifiers were then integrated: K-nearest neighbor (KNN), support vector machine (SVM) with radial basis function, and Random forest classifiers, all to recognize people $[12,13,14]$. Before integrating machine learning classifiers feature extraction is one of the important steps because the learning process and accuracy of machine learning (ML) classifiers depend on it [15]. If there are no unique recognition features in the dataset then machine learning classifiers cannot produce better results. On the other hand, in artificial intelligence and deep learning, manual feature extraction from the signal is not required because the model itself extracts unique features during training [15]. In other words, integrating the ML than AI models also helps to better understand the signal parameters.

\section{Example of results}


In this section, a few representative results are presented in the context of breathing diversity based on unique identification and monitoring of vital signs. Figure 3 illustrates the experimental scenario and Figure 4 shows some of the results of the experiment. Figure 4 (a) shows there is significant variation in inhaling and exhale area ratio for two different participants. As mentioned before, a trapezium within 30\%-70\% of the amplitude of the radar captured respiration signal was used to calculate the area within that segment and to calculate area ratios. Figure 4 (b) illustrates that after performing physiological activities, subject exhale areas are significantly different for each participant. As different people intake different amounts of air there is significant variation in their airflow profile. After collecting all these respiratory dynamics-related features, they were put as a matrix into the machine learning classifier for recognizing different participants. It should be mentioned that in our experiment we considered both normal breathing dynamics and breathing dynamics captured after performing brief exertions. Fig. 4 (c) illustrates that the confusion matrix where the diagonal
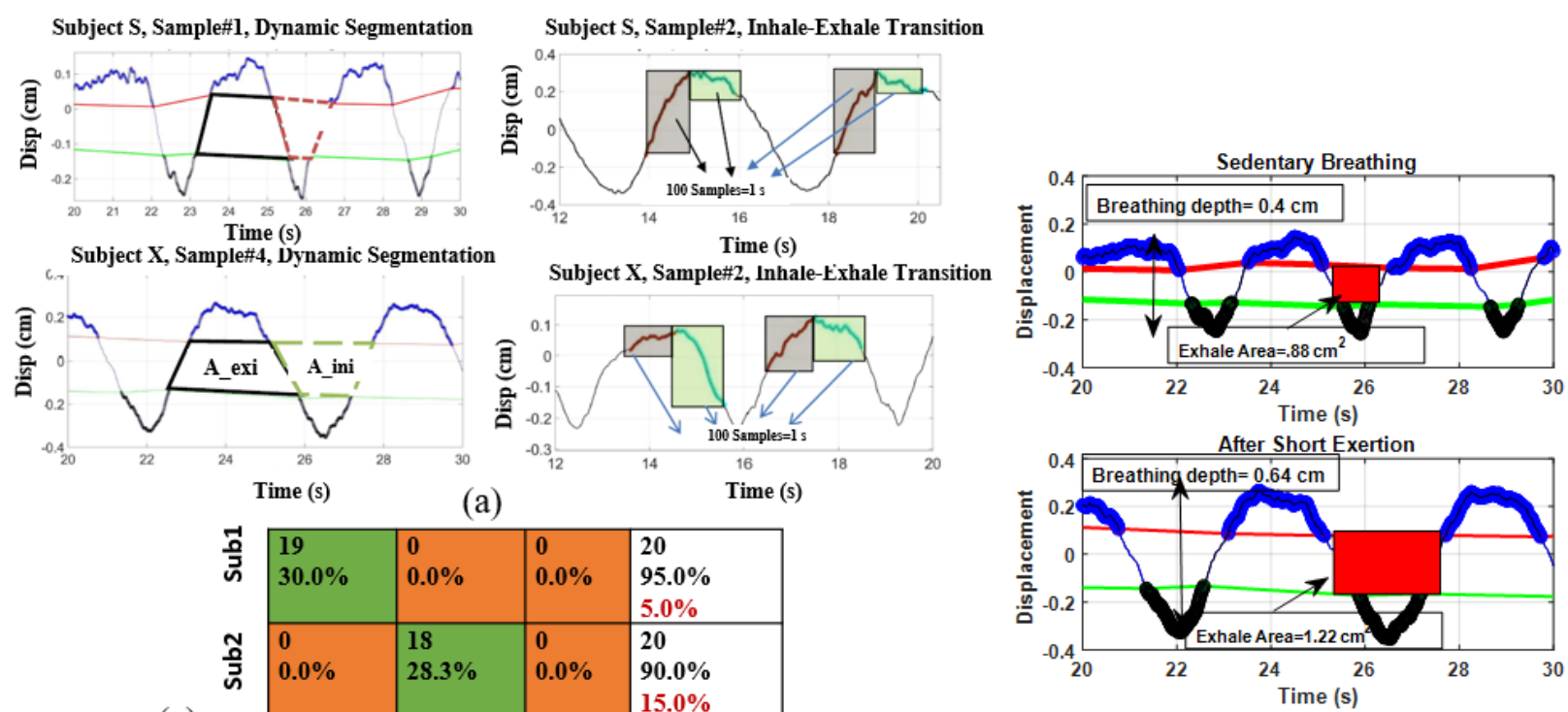

(c)

\begin{tabular}{|c|c|c|c|c|}
\hline 年 & $\begin{array}{l}19 \\
30.0 \%\end{array}$ & $\begin{array}{l}0 \\
0.0 \%\end{array}$ & $\begin{array}{l}0 \\
0.0 \%\end{array}$ & $\begin{array}{l}20 \\
95.0 \% \\
5.0 \%\end{array}$ \\
\hline กัก & $\begin{array}{l}0 \\
0.0 \%\end{array}$ & $\begin{array}{l}18 \\
28.3 \%\end{array}$ & $\begin{array}{l}0 \\
0.0 \%\end{array}$ & $\begin{array}{l}20 \\
90.0 \% \\
15.0 \% \\
\end{array}$ \\
\hline 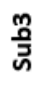 & 1 & $\begin{array}{l}2 \\
3.3 \%\end{array}$ & $\begin{array}{l}17 \\
26.7 \%\end{array}$ & $\begin{array}{l}20 \\
85 \% \\
20.0 \% \\
\end{array}$ \\
\hline$\frac{\overline{0}}{\overline{0}}$ & $\begin{array}{l}20 \\
95.0 \% \\
10.0 \%\end{array}$ & $\begin{array}{l}20 \\
90.0 \% \\
10.0 \% \\
\end{array}$ & $\begin{array}{l}20 \\
85.0 \% \\
15.0 \%\end{array}$ & $\begin{array}{l}60 \\
90.0 \% \\
15.0 \% \\
\end{array}$ \\
\hline
\end{tabular}

(b)

Fig. 4. Subject identification. Breathing diversity for two different participants is illustrated through their inhale/exhale area ratios and dynamics variations in the transition from inhale to exhale (a). Two different participant exhale area changes can be seen to change significantly after performing breif exertion (b). Confusion matrix is shown for five participants with a classification accuracy of around $92 \%$ even after mixing up sedentary breathing and breathing after performing brief exertion. Taken from [12,13]. 
column represents the success rate of recognizing people. From Fig. 4 (c) the results for three participants have a success rate for the combined mixtures of around $90 \%$, and just for sedentary breathing alone, it was around 95\%. The classification accuracy indicates that the proposed system has potential benefits for biometric identification systems.

Other research groups also demonstrated the efficacy of this technology, with some using heart-based dynamics, like a cardiac cycle, for different participants. After capturing radar-respiration signals they took five different points within the segment of inhaling and exhale [6,9]. In between five different points, they calculated the time and area which acts as a unique feature. Using these heart-based dynamic features they also integrated a machine learning classifier to recognize people from their heart-based geometry. Over the last five years, there is a significant improvement in terms of accuracy of recognizing people from their breathing dynamics [11]. In our most recent reported research, we also proposed a new improved version of the dynamic segmentation algorithm to improve accuracy and our reported results are still under review [16, 17]. In the improved version of the dynamic segmentation algorithm, we integrated both the heart and respiratory-based dynamics by introducing peak search and triangulation methods together. Previously reported dynamic segmentation algorithm considers the $30 \%-70 \%$ amplitude of the signal so it excluded the heart-beat related information as heartbeat information lies in between the inhale and exhale transition [10, 16]. For the newly proposed algorithm, we have created an array of maxima and minima of the signal and then constructed a triangle between three points (two consecutive maxima and one minimum) and calculated the area of the triangle for inhaling and exhale episodes. Then we extracted the average of inhaling and exhaling areas within a window of 12 seconds [16]. We selected a 12 seconds window based on the FFT window size sampling limit and within a particular window, there should be multiple complete breathing cycles $[16,17]$. Integrating the peaks helps to consider the effect of cardiac dynamics, where the inhale area illustrates ventricular filling and the exhale area represents ventricular contraction. Figure 5 illustrates the inhale and exhale area differences within radar captured respiration patterns for six different participants. This improved version of the algorithm significantly increased the accuracy of the system both for sedentary 
and post-physiological activity breathing [16]. Continued experimentation and further exploration are required to bring this sensor technology into the practical real-world of implementation.

\section{Challenges of non-contact identity authentication technology}

Advancements in artificial intelligence and machine learning algorithms enable radar non-contact identity authentication technology to extract and analyze relevant identity features, including the biometric
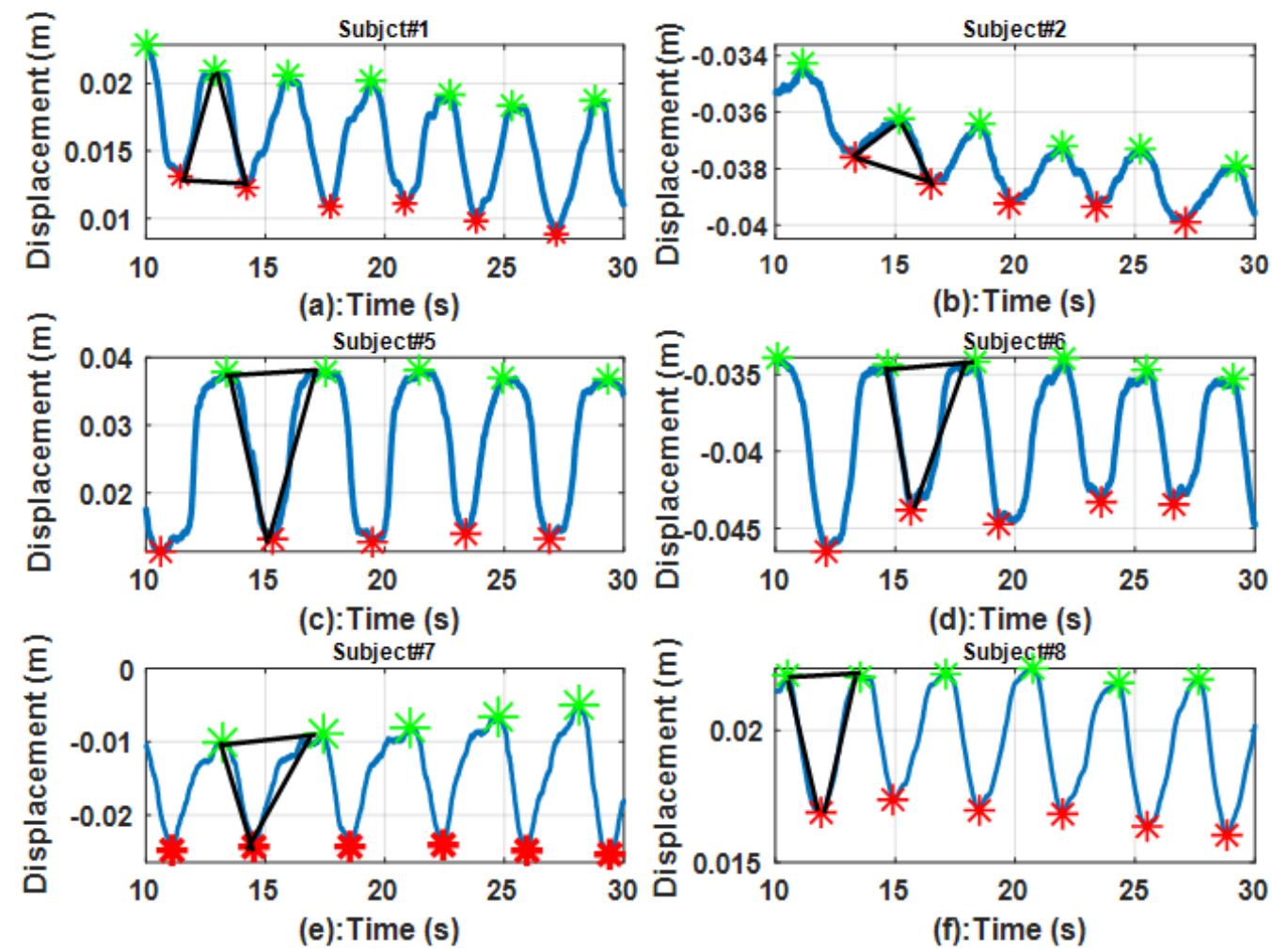

Fig. 5. Extracted respiratory features for six different participants: (a) subject-1, (b) subject-2, (c) subject-3, (d) subject-4, (e) subject-5, and (f) subject-6. From the radar-captured signals, it is quite clear that the inhale and exhale areas based on heartbeat peaks of the signal differ significantly. From [16].

characteristic of the cardiac signal [11]. However, there remain significant challenges that need to be overcome to bring this technology into practice. One of the challenges researchers are investigating is the implementation of this sensor technology in multi-subject environments [18]. Because in real life, especially in airports or in-home environments, there is a probability of the presence of multiple subjects in front of the radar. When there are multiple subjects present, a combined mixture of breathing patterns results, from which 
it is difficult to separate individual respiration patterns. Researchers have already demonstrated some integrated signal processing techniques to isolate respiratory patterns from a combined mixture of breathing patterns [18], and continuous identity authentication in multi-subject scenarios remains under active investigation. Figure 6 illustrates current research challenges for continuous identity authentication in multiple subject environments.

Another significant challenge is the development of robust unique feature extraction algorithms, as respiratory patterns may change due to physical activities or emotional stresses. Researchers are also investigating ways to find a unique feature for different physical activities and emotional stresses. To make

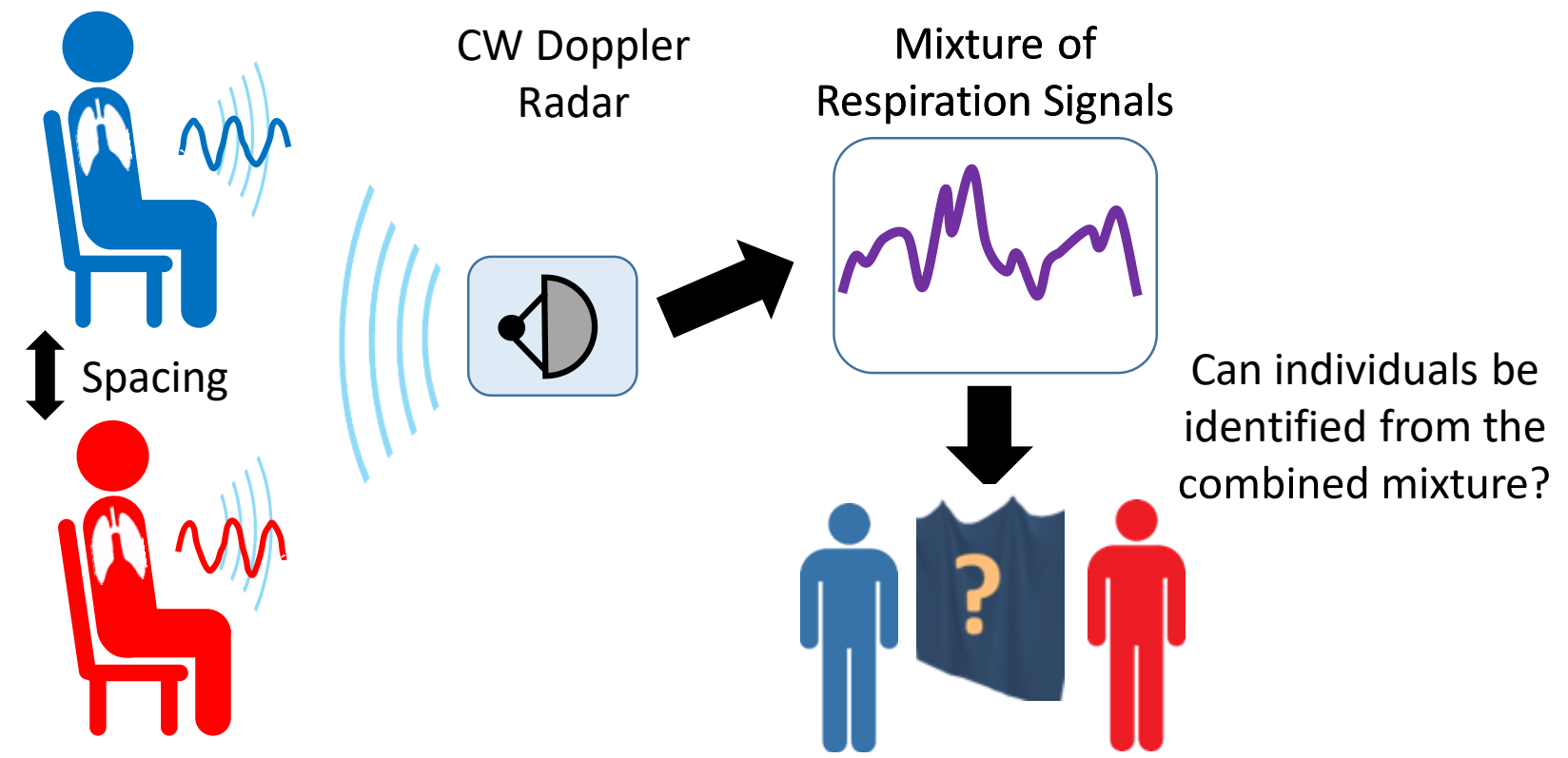

Fig. 6. Challenges for recognizing multiple people from radar-captured combined respiration patterns. Robust separation of independent respiratory signatures and recoginition of distinct patterns associated with individuals remains an important topic of research. Based on [18].

this technology practical, robust unique feature extraction algorithms must be developed and this has led some to integrate artificial intelligence.

If respiration patterns are going to be as future passwords, there is a need to investigate the integration of cryptographic mechanisms with radar-captured respiration patterns [17]. By integrating security protocols and cryptographic mechanisms, data breaches or any other fraud activities can be stopped [17]. To improve the security of the entire system further investigation continues and additional experiments are required. 
Ongoing research within the radar community aims to validate this non-contact respiration-based identity authentication technology for more realistic scenarios for a multi-subject environment as well as integrating security protocol with the system. Outstanding challenges include investigating how robust the unique identification algorithms for identity authentication are for different emotional stresses or different physiological activities.

\section{Conclusion}

Over the past four decades, there has been significant improvement in utilizing short-range radar for healthcare applications. In this article, a potential new role of radar in biometric identification is discussed which can improve on vulnerabilities in traditional identity authentication systems. Current biometric modalities such as fingerprints, retina scans, and facial recognition, limit the effectiveness of remote or unattended authentication applications. Researchers are investigating the integration of new signal processing techniques and novel receiver architectures to bring radar sensor authentication technology into real-world implementations. The ability to unobtrusively identify or monitor human subjects remotely using radar sensed respiration data is of growing importance in a wide variety of applications.

Experimental results for radar-captured respiration patterns for recognizing human subjects have also been discussed. Innovative means for collection and full understating of these results will be key to developing truly useful unique identification algorithms for identifying people unobtrusively. With the advancement of machine learning and artificial intelligence, integrated Doppler radar respiration sensing technologies will revolutionize the way we monitor/identify ourselves and the world around us and offer new applications in human-machine interaction.

\section{REFERENCES:}

[1] R. Watson-Watt, "Radar in War and in Peace", Nature, vol. 155, pp. 319-324,1945

[2] J. C. Lin, "Microwave Sensing of Physiological movement and volume change: a review," Bioelectromagnetics., vol. 13, no. 6, pp. 557-565, 1992, doi: 10.1002/bem.2250130610.

[3] G. Benchetrit, "Breathing pattern in humans: diversity and individuality," Respiration Physiology, vol. 122, no. 2-3, pp. 123-129, Sep. 2000, doi: 10.1016/S0034-5687(00)00154-7. 
[4] C. Li, V. M. Lubecke, O. Boric-Lubecke, and J. Lin, "A Review on Recent Advances in Doppler Radar Sensor for Non-contact Healthcare Monitoring”, IEEE Trans. Microwave Theory Techn., vol. 61, non. 5, pp. 2046-2060, May 2013, doi: 10.1109/TMTT.2013.2256924.

[5] "Chaos Computer Club (CCC), Chaos Computer club breaks Apple touch ID. https://wwww.ccd.de/en/updates/2013/ccc-breaks-apple-touchid, September 2013, Accessed by November 2016." [Online]. Available: https://wwww.ccd.de/en/updates/2013/ccc-breaks-appletouchid, September 2013.

[6] F. Lin, C. Song, Y. Zhuang, W. Xu, C. Li, and K. Ren, "Cardiac Scan: A Non-contact and Continuous Heart-based User Authentication System," in Proceedings of the 23rd Annual International Conference on Mobile Computing and Networking - MobiCom '17, Snowbird, Utah, USA, 2017, pp. 315-328, doi: 10.1145/3117811.3117839.

[7] A. Droitcour, V. Lubecke, Jenshan Lin, and O. Boric-Lubecke, "A microwave radio for Doppler radar sensing of vital signs," in 2001 IEEE MTT-S International Microwave Symposium Digest (Cat. No.01CH37157), Phoenix, AZ, USA, 2001, vol. 1, pp. 175-178, doi: 10.1109/MWSYM.2001.966866.

[8] A. Droitcour, "Non-Contact Measurement of Heart and Respiration Rates with a Single-Chip Microwave Doppler Radar", Ph.D. dissertation, Stanford University, June 2006.

[9] K. Shi, C. Will, R. Weigel, and A. Koelpin, "Contactless person identification using cardiac radar signals," in 2018 IEEE International Instrumentation and Measurement Technology Conference (I2MTC), Houston, TX, May 2018, pp. 1-6, doi: 10.1109/I2MTC.2018.8409645.

[10] A. Rahman, V. M. Lubecke, O. Boric-Lubecke, J. H. Prins, and T. Sakamoto, "Doppler Radar Techniques for Accurate Respiration Characterization and Subject Identification," IEEE J. Emerg. Sel. Topics Circuits Syst., vol. 8, no. 2, pp. 350-359, Jun. 2018, doi:

10.1109/JETCAS.2018.2818181.

[11] S. M. M. Islam, O. Borić-Lubecke, Y. Zheng, and V. M. Lubecke, "Radar-Based Non-Contact Continuous Identity Authentication," Remote Sensing, vol. 12, no. 14, p. 2279, Jul. 2020, doi: $10.3390 /$ rs 12142279 .

[12] S. M. M. Islam, A. Sylvester, G. Orpilla, and V. M. Lubecke, "Respiratory Feature Extraction for Radar-Based Continuous Identity Authentication," in 2020 IEEE Radio and Wireless Symposium (RWS), San Antonio, TX, USA, Jan. 2020, pp. 119-122, doi: 10.1109/RWS45077.2020.9050013.

[13] S. M. M. Islam, A. Rahman, N. Prasad, O. Boric-Lubecke and V. M. Lubecke, "Identity Authentication System Using Support Vector Machine on Radar Respiration Measurement," in Proc. 93 ${ }^{\text {rd }}$ ARFTG Microwave Measurement Conference (ARFTG'19), Boston, MA, USA, Jun. 2019, pp. 1-5, doi: 10.1109/ARFTG.2019.8739240.

[14] S. M. M. Islam, A. Rahman, E. Yavari, O. Boric-Lubecke and V. M. Lubecke, "Identity Authentication of OSA Patients Using Microwave Doppler Radar and Machine Learning Classifiers," in 2020 IEEE Radio and Wireless Symposium (RWS), San Antonio, TX, USA, Jan. 2020, pp. 251254, doi: 10.1109/RWS45077.2020.9049983.

[15] A. V. Joshi, "Machine Learning and Artificial Intelligence”, Springer Nature Publishing, 2019.

[16] S. M. M. Islam, and V. M. Lubekce, "Non-Contact Continuous Identity Authentication in Multiple Subject Environments," IEEE Transaction on Microwave Theory and Technique (Submitted).

[17] S. M. M. Islam, Y. Zheng, O. Boric-Lubecke, and V. M. Lubecke, "Modality-Switch Compliance Tracking for Unobtrusive Sleep Apnea Test", IEEE Transaction on Mobile Computing. (Submitted).

[18] S. M. M. Islam, O. Boric-Lubecke, and V. M. Lubekce, "Concurrent Respiration Monitoring of Multiple Subjects by Phase-Comparison Monopulse Radar Using Independent Component Analysis (ICA) with JADE Algorithm and Direction of Arrival (DOA)," IEEE Access, pp. 1-1, 2020, doi: 10.1109/ACCESS.2020.2988038. 
SHEKH M. M. ISLAM (Member, IEEE) received the B.Sc. (Hons) and M. Sc degree in electrical and electronic engineering from the University of Dhaka, Dhaka, Bangladesh in 2012 and 2014 , respectively. He received his Ph.D. degree in electrical engineering from the University of Hawaii at Manoa, Honolulu, HI, the USA, with a focus on biomedical applications incorporating RF/Microwave technologies in December 2020. From 2014 to 2016, he worked as a Lecturer with the Electrical and Electronic Engineering Department, University of Dhaka. His research interests include radar systems, antenna array signal processing, adaptive filter technique, and machine learning classifiers for pattern recognition. In Summer 2019, he also worked as a Radar System and Applications Engineering Intern with ON Semiconductor, Phoenix, AZ, USA.

Dr. Islam is also an active member of the IEEE Microwave Theory and Technique (MTT) and the IEEE Engineering in Medicine and Biology (EMBS) Society. He was a recipient of the 2020 University of Hawaii at Manoa Department of Electrical Engineering Research Excellence Award. He was also the student paper Finalist in IEEE Radio Wireless Week (RWW'19) Conference, which was held in FL, USA.

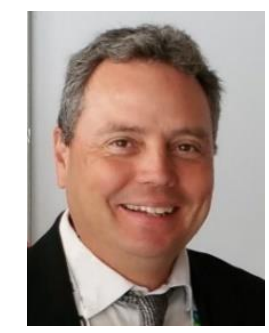

Victor M. Lubecke (Fellow, IEEE) received his M.S. and Ph.D. degrees in electrical engineering from the California Institute of Technology, Pasadena, in 1990 and 1995, respectively, and his B.S.E.E. degree from the California Polytechnic Institute, Pomona, in 1986. Dr. Lubecke is currently a Professor of Electrical Engineering at the University of Hawaii, Manoa, HI, USA. From 1998 to 2003, Dr. Lubecke was with Bell Laboratories, Lucent Technologies, where his research focused on remote sensing technologies for biomedical and industrial applications, and on microelectromechanical systems (MEMS) and 3-D wafer-scale integration technologies for wireless and optical communications. From 1987 through 1996, he was with the NASA Jet Propulsion Laboratory (JPL), and from 1996 to 1998, he was with the Institute for Physical and Chemical Research (RIKEN), Sendai, Japan, where his research involved terahertz and MEMS technologies for space remote sensing and communications applications.

Prof. Lubecke is an IEEE Fellow, an emeritus Distinguished Microwave Lecturer ('06-'08) of the IEEE Microwave Theory and Techniques Society and served as Topic Editor for the IEEE Transactions on Terahertz Science and Technology. He served as Vice-Chair for the 2017 IEEE IMS, and on Technical and Steering Committees for various IEEE and SPIE symposia. He was the recipient of the 2000 Microwave Prize for the best paper presented at the Asia-Pacific Microwave Conference. He also co-authored student competition papers, which were selected for First Place at the 2003 IEEE MTT-S International Microwave Symposium (IMS), Third Place at the 2001 IEEE Engineering in Medicine and Biology Society (EMBS) Conference, and honorable mention at both the 2001 IEEE MTT-S IMS and the 2006 IEEE Radio Wireless Symposium (RWS). He was co-recipient of the Emerging Technology Award at TechConnect 2007, cofounded two technology start-up companies, and holds 7 U.S. patents with several more pending. His current research interests include remote sensing technologies, biomedical sensors, animal tracking technology, MEMS, heterogeneous integration, and microwave/terahertz radio. 